\title{
Observational Study Protocol Version Time Perspective Code
}

National Cancer Institute

\section{Source}

National Cancer Institute. Observational Study Protocol Version Time Perspective Code. NCl Thesaurus. Code C93868.

A coded value specifying the temporal relationship of observation period to time of subject enrollment. 